\title{
Rotation as a source of asymmetry in the double-peak lightcurves of the bright EGRET pulsars
}

\author{
J. Dyks ${ }^{1}$ and B. Rudak ${ }^{1,2}$ \\ ${ }^{1}$ Copernicus Astronomical Center, Toruń, Poland \\ 2 Dept. of Astronomy and Astrophysics, Nicholas Copernicus University, Toruń, Poland
}

Received 10 December 2001 / Accepted 4 July 2002

\begin{abstract}
We investigate the role of rotational effects in inducing asymmetry present above $\sim 5 \mathrm{GeV}$ in the double-peak lightcurves of the bright EGRET pulsars: Vela, Crab, and Geminga. According to Thompson (2001), the trailing peak dominates over the leading peak above $\sim 5 \mathrm{GeV}$ consistently for all three pulsars, even though this is not the case over the entire energy range of EGRET, i.e. above $\sim 100 \mathrm{MeV}$. We present the results of Monte Carlo simulations of electromagnetic cascades in a pulsar magnetosphere within a single-polar-cap scenario with rotationally-induced propagation effects of the order of $\beta$ (where $\beta$ is the dimensionless local corotation velocity). We find that even in the case of nearly aligned rotators with spin periods of $P \sim 0.1$ s rotation may lead to asymmetric (with respect to the magnetic axis) magnetic photon absorption which in turn leads to asymmetric gamma-ray pulse profiles. The resulting features - softer spectrum of the leading peak and the dominance of the trailing peak above $\sim 5 \mathrm{GeV}$ - agree qualitatively with the EGRET data of the bright gamma-ray pulsars.
\end{abstract}

Key words. stars: rotation - stars: pulsars: general

\section{Introduction}

Good quality gamma-ray data for three pulsars - Vela, Crab, and Geminga - acquired with EGRET aboard the CGRO tempts to analyse the properties of pulsar high-energy radiation as a function of photon energy and phase of rotation. Gammaray spectra of pulsed radiation from these sources (as well as from three other EGRET pulsars: B1706-44, B1951+32, and B1055-52) extend up to $\$ 10 \mathrm{GeV}$. All three pulsars feature gamma lightcurves characterised by two strong peaks separated by 0.4 to 0.5 in rotational phase. These double-peak pulses are asymmetrical and their profiles change with energy. Above $\sim 100 \mathrm{MeV}$ the leading peak (LP) is stronger than the trailing peak (TP) in the case of the Vela and the Crab pulsars, and the opposite is true for Geminga. However, for all three pulsars their leading peaks exhibit lower energy cutoffs - around $\sim 5 \mathrm{GeV}$ - than the trailing peaks (TP). In other words, the trailing peaks dominate over the leading peaks above $\sim 5 \mathrm{GeV}$ (Thompson 2001). In the case of the Vela pulsar and Geminga, this effect is accompanied by the softening of the spectrum of the leading peak (Fierro et al. 1998; Kanbach 1999). The potential importance of the double-peak pulse asymmetry in the case of Vela was already acknowledged - at the time when the COSB data became available - by Morini (1983) who attempted to explain the asymmetry with a hybrid model, with two different mechanisms responsible for the formation of the leading and the trailing peak.

Send offprint requests to: J. Dyks, e-mail: jinx@ncac.torun.pl
High-energy cutoffs in pulsar spectra are interpreted within polar cap models as due to one-photon absorption of gammarays in strong magnetic field with subsequent $e^{ \pm}$-pair creation. A piece of observational support for such an interpretation comes from a strong correlation between the inferred "spindown" magnetic field strength and the position of the highenergy cutoff (Baring \& Harding 2000; Baring 2001). This in turn opens a possibility that the observed asymmetry between LP and TP, i.e. the dominance of LP over TP above $\sim 5 \mathrm{GeV}$, is a direct consequence of propagation effects (which eventually lead to stronger magnetic photon absorption for photons forming LP than TP) rather than due to some inherent property of the gamma-ray emission region itself.

The aim of this paper is to investigate the role of pulsar rotation in a built-up of such asymmetry in the double-peak pulse profiles. We consider purely rotational effects: due to presence of rotation-induced electric field $\boldsymbol{E}$, aberration of photon direction and slippage of magnetosphere under the photon's path. In Sect. 2 we compare them with some other effects which may be responsible for the asymmetry (like various distortions of the magnetic field structure). In Sect. 3 we show that the rotation effects result in an asymmetric pair production rate for the leading and the trailing part of the magnetosphere even in the case when the magnetic field structure and the population of radiating particles are symmetric around the magnetic pole. In Sect. 4 asymmetric pulse profiles are calculated as a function of photon energy and then the model predictions of the ratio of fluxes in the leading and trailing peaks are compared with the 
inferred ratio for Vela at different energy bins. In Sect. 5 we address the significance of rotation-driven asymmetry across the pulsar parameter space. Our main results are discussed in Sect. 6.

\section{Symmetric features of the model}

It has been argued in many studies of radio properties of pulsars (e.g. Blaskiewicz et al. 1991; Gangadhara \& Gupta 2001) that a rigidly rotating static-like dipole can be used as a good approximation of dipolar magnetic field as long as only most important rotation effects, of the order of $\beta=v / c$ (where $v$ is the local corotation velocity and $c$ is the speed of light), are to be considered. According to order-of-magnitude estimates, small distortions of the dipolar magnetosphere induced by rotationally-driven currents can be neglected: longitudinal currents suspected to flow within the open field line region cannot modify $\boldsymbol{B}$ by a factor exceeding $\beta^{3 / 2}$ whereas toroidal currents due to plasma corotation change $\boldsymbol{B}$ barely by a value of the order of $\beta^{2}$. A more comprehensive discussion of the influence of currents on the magnetospheric structure can be found in Beskin (1999) and references therein.

Below we follow the approximation of a rigidly rotating static-like dipole: at any instant the magnetic field has the shape of a static dipole in the frame which corotates with a neutron star. Moreover, the magnetosphere is assumed to be filled out everywhere with the Goldreich-Julian charge density, so that a rotation-induced electric field $\boldsymbol{E}=-\boldsymbol{\beta} \times \boldsymbol{B}$ is present in the OF, whereas $\boldsymbol{E}^{\prime}=0$ in the frame corotating with the star. We neglect deviations from this corotational electric field which are present in the charge-deficient polar gap region - they do not exceed a factor of $\beta^{3 / 2}$.

In our Monte Carlo simulations (Sect. 4) development of gamma-ray radiation is based on the model of Daugherty \& Harding (1982), with primary electrons being injected along magnetic field lines at an altitude of a few neutron star radii, at the magnetic colatitude corresponding to the last open magnetic field lines, and uniformly in the magnetic azimuth. The electrons are assumed to accelerate instantly to the energy $E_{0} \sim 10^{7} \mathrm{MeV}$ and subsequently to cool down emitting curvature photons. Some of the photons induce in turn electromagnetic cascades which propagate outwards in a form of a hollow cone beam (see Dyks \& Rudak 2000 for detailed description of directionality aspects of the casades as well as viewing geometry).

\section{Rotation-driven asymmetry}

In this section we present in detail the mechanisms which lead to rotation-induced asymmetry in the (otherwise axially symmetric) hollow-cone gamma-ray beam. For the sake of better demonstration of the effects we consider an exaggerated case: propagation of a photon in the equatorial plane of a fast orthogonal rotator. Figure 1 shows the case for the rotation period $P=1.5 \mathrm{~ms}$ : photons of the same energy in the corotating frame (CF) are emitted from two opposite points on the outer rim of the polar cap tangentially to the magnetic field lines in the CF. To follow their straight-line propagation in the inertial observer

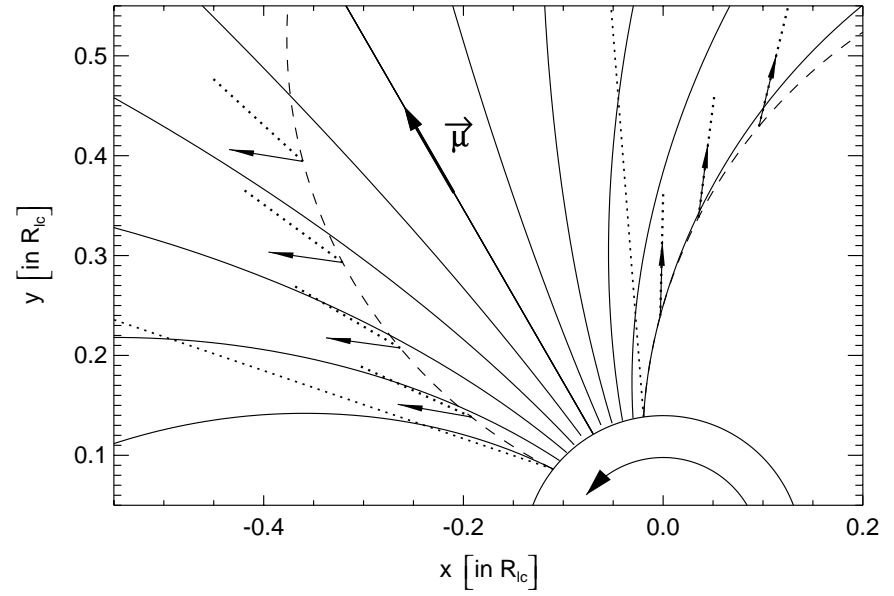

Fig. 1. Top view of orthogonally rotating pulsar with the spin period $P=1.5 \mathrm{~ms}$; the sense of rotation is counter-clockwise (indicated); the cartesian coordinates are in units of the light cylinder $R_{\mathrm{lc}}=c P / 2 \pi$. The magnetic field lines are approximated with the static-like dipole of the magnetic moment $\boldsymbol{\mu}$ (indicated). Two photon trajectories (starting from two opposite points on the polar cap) in the inertial observer frame (OF) are marked with two long dotted lines. These are the prototypes of the leading peak (on the left) and the trailing peak (on the right). In the corotating frame (CF) these trajectories follow the long dashed lines. Short segments of the dotted-line trajectories (OF) have been transformed via rotation to several actual photon locations in the magnetosphere, and thus they indicate the local propagation directions $\hat{\eta}$ of the photons in OF. Solid arrows at these locations point along the directions $\hat{\eta}^{\mathrm{FP}}$ of local free propagation in OF. Note much larger angles between $\hat{\eta}^{\mathrm{FP}}$ and $\hat{\eta}$ for the photons of the leading peak than for the photons of the trailing peak.

frame (OF) three effects have to be taken into account: 1) In the OF the photons propagate at an aberrated direction (dotted lines in Fig. 1) and differ in energy. 2) The rotation-induced electric field $\boldsymbol{E}=-\boldsymbol{\beta} \times \boldsymbol{B}$, which is present in the OF, modifies the rate of the magnetic photon absorption in a different way for photons forming the LP than for photons forming the TP. 3) The electromagnetic field in the OF is time-dependent: because of the rotation the photons propagate through different parts of the magnetosphere.

We find that the second effect - due to rotationally induced $\boldsymbol{E}$ - plays a dominant role in generating the asymmetry in the magnetic absorption rate $R$ between photons of the LP and the TP. An importance of a weak electric field $\boldsymbol{E} \perp \boldsymbol{B}$ for the rate $R$ was for the first time recognized by Daugherty \& Lerche (1975) who presented also its quantitative treatment. A consequence of its presence is that the rate $R$ does not vanish along the direction of local $\boldsymbol{B}$ any more, and becomes non-axisymmetric with respect to it as well. Instead, the rate $R$ vanishes along two new directions which lie in the plane perpendicular to $\boldsymbol{E}$ and deviate from $\boldsymbol{B}$ by angle $\sim E / B$ in such a way that in a local coordinate frame with $\hat{z}\|\boldsymbol{B}, \hat{y}\| \boldsymbol{E}$, and $\hat{x} \| \boldsymbol{E} \times \boldsymbol{B}$ the "free propagation" direction $\hat{\eta}^{\mathrm{FP}}=\left[\eta_{x}^{\mathrm{FP}}, \eta_{y}^{\mathrm{FP}}, \eta_{z}^{\mathrm{FP}}\right]$ has two solutions: $\left[E / B, 0, \pm\left(1-E^{2} / B^{2}\right)^{1 / 2}\right]$. For definiteness, hereafter we will consider photons which propagate outwards within the regions above the northern magnetic pole (i.e. with propagation vectors $\hat{\eta}$ satisfying $\hat{\eta} \cdot \boldsymbol{B}>0$ ) which corresponds to the 
case of $\eta_{z}^{\mathrm{FP}}=+\left(1-E^{2} / B^{2}\right)^{1 / 2}$. Figure 2 shows the mean free path $\lambda_{\mathrm{mfp}}=c / R$ for the magnetic photon absorption for different directions in the plane perpendicular to $\boldsymbol{E}$. The rate $R$ was calculated by performing Lorentz transformation to a frame in which $\boldsymbol{E}^{\prime}=0$ and then applying a purely magnetic formula. The formula of Erber (1966) with a modification of Daugherty \& Harding (1983) correcting its near-threshold inaccuracy is used throughout the paper:

$R^{\prime}\left(\varepsilon^{\prime}, \boldsymbol{B}^{\prime}, \sin \theta_{B}^{\prime}\right)=c_{1} \sin \theta_{B}^{\prime} B^{\prime} \exp \left[-c_{2} f /\left(\varepsilon^{\prime} \sin \theta_{B}^{\prime} B^{\prime}\right)\right]$,

where $\theta_{B}^{\prime}=\angle\left(\hat{\eta}^{\prime}, \boldsymbol{B}^{\prime}\right), \varepsilon^{\prime}$ is the photon energy; $c_{1}$ and $c_{2}$ are constant quantities, while $f=f\left(\varepsilon^{\prime}, \boldsymbol{B}^{\prime}\right)$ is the near-threshold correction of Daugherty \& Harding (1983). Six lines in Fig. 2 correspond to six values of the ratio $E / B: 0.01,0.1,0.2,0.5$, 0.9 , and 0.999 . Note that the free propagation direction deviates from the local $\boldsymbol{B}$ by angle $\theta^{\mathrm{FP}}$ which increases with increasing contribution of the electric field: $\theta_{z}^{\mathrm{FP}} \equiv \arccos \left(\eta_{z}^{\mathrm{FP}}\right)=$ $\arcsin (E / B) \simeq E / B$. Moreover, $R$ increases $\left(\lambda_{\mathrm{mfp}}\right.$ decreases $)$ monotonically with increasing angle $\angle\left(\hat{\eta}, \hat{\eta}^{\mathrm{FP}}\right)$. As long as $E \ll$ $B$, magnetic photon absorption rate $R$ remains approximately symmetric around the free propagation direction $\hat{\eta}^{\mathrm{FP}}$.

The directions of $\hat{\eta}^{\mathrm{FP}}$ at various points within the magnetosphere are shown in Fig. 1 in the OF (with solid arrows). At the points of photon emission the direction $\hat{\eta}^{\mathrm{FP}}$ overlaps with the photon direction $\hat{\eta}$ since this is where the angle $\theta_{B}$ between the photon direction and the magnetic field line equals $\theta_{B} \simeq E / B$ and consequently $R=0$ (Harding et al. 1978; Zheng et al. 1998). Initially, therefore, the rate $R$ is the same for photons emitted from two opposite sites of the polar cap rim which in this picture give rise to the leading peak and the trailing peak. But as the photons propagate outward their $\hat{\eta}$ begin to deviate from local free propagation directions $\hat{\eta}^{\mathrm{FP}}$ opening thus a possibility for magnetic photon absorption and electron-positron pair creation. Three effects are responsible for it to happen: (1) curvature of the magnetic field lines, (2) increase of $E / B$ with altitude, and (3) slippage of magnetic field lines under the photon's path. For a static-like dipole (assumed in Sect. 2) the curvature of lines is symmetric with respect to the magnetic axis. Consequently, symmetry is expected between the rates of magnetic absorption for LP photons and TP photons. Any asymmetry between the LP and the TP may occur via the effects (2) and (3) only. Whenever the curvature of magnetic field lines happens to be relatively large (which is the case at the polar cap rims of pulsars with $P \sim 0.1 \mathrm{~s}$ ) the effect (1) significantly dominates over (2) and (3), which means that the resulting asymmetry is quite subtle. However, for a relatively small curvature of magnetic field lines (and appreciable local corotation velocities $\beta$ ) the resulting asymmetry becomes pronounced [note that these "favourable" conditions are fulfilled within outer-gap accelerators].

We find that the effect (2) - the presence of electric field - is crucial for generating the asymmetry. The way in which this effect operates can be most clearly assessed by inspecting Fig. 2. Let us consider a photon propagation vector $\hat{\eta}$ anchored at the origin of frame in the figure. For photons emitted close to the star, both in the LP and the TP, $\hat{\eta}$ initially points along the $\hat{\eta}^{\mathrm{FP}}$ direction which differs only slightly from the direction of $\boldsymbol{B}$ in Fig. 2 since $E \ll B$. As the LP photon moves away

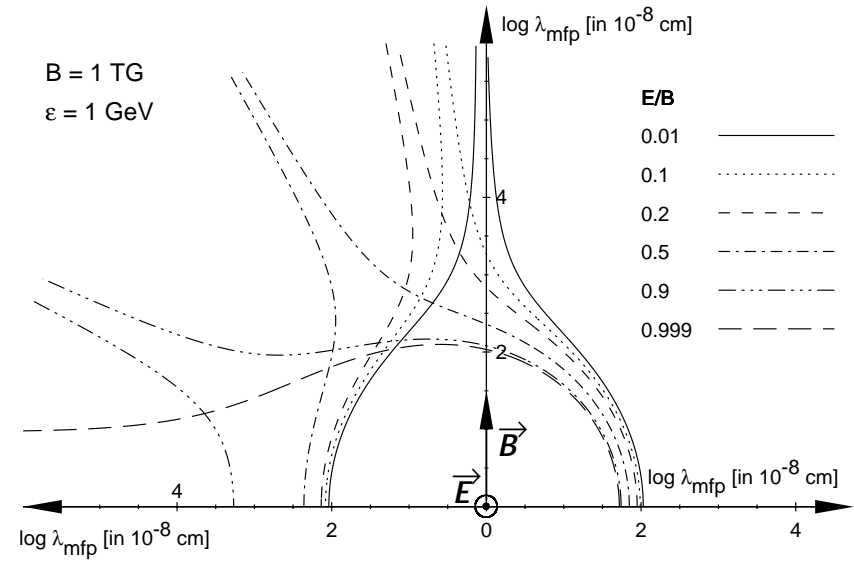

Fig. 2. Directional dependence of the mean free path $\lambda_{\mathrm{mfp}}$ for magnetic absorption of a photon emitted at the center of the reference frame (with the coordinates $[0,0]$ ) is shown for six values of $E / B$ (denoted with six different line types). The electric field vector $\boldsymbol{E}$ is pointing at the right angle to the page, and toward the reader; the $\boldsymbol{E} \times \boldsymbol{B}$ vector is parallel to the horizontal axis, and pointing to the left. The mean free path $\lambda_{\mathrm{mfp}}$ is symmetric with respect to the horizontal axis.

from emission point, its propagation vector rotates clockwise in Fig. 2 which reflects the fact that $\boldsymbol{B}$ diverges from $\hat{\eta}$ due to the magnetic field line curvature. At the same time, however, the directional pattern of $\lambda_{\mathrm{mfp}}$ rotates counterclockwise due to increase in $E / B$ which additionally enhances the absorption rate. In the case of the TP photon, however, both its propagation vector $\hat{\eta}$ and the directional pattern of $\lambda_{\text {mfp }}$ rotate in the same direction (counterclockwise) with respect to $\boldsymbol{B}$ in Fig. 2 so that the absorption rate is weakened. Thus, for photons within the LP the effects (1) and (2) cummulate, whereas for the TP they effectively tend to cancel out each other, and the expected asymmetry between the peaks is due to stronger absorption suffered by photons within the LP than by photons of the same energy within the TP. Accordingly, the high energy cutoff in the LP spectrum will occur at a slightly lower energy than the cutoff in the TP spectrum. The slippage (3) affects this picture in the way which depends on both the rotation period and the photon position within the magnetosphere, but an overall picture remains unchanged. For most rotation periods $(P>$ a few $\mathrm{ms}$ ), the slippage reduces the asymmetry only marginally. For the fastest rotators $(P \sim 1.5 \mathrm{~ms})$ it enhances the asymmetry by making photons of the TP to propagate along the free propagation direction (or, equivalently, along the magnetic field lines in the CF). The latter case is presented in Fig. 1 where the photon propagation direction $\hat{\eta}$ as seen in the OF (dotted lines) and the local free propagation direction $\hat{\eta}^{\mathrm{FP}}$ in the OF (solid arrows) are shown for several positions along the photon trajectory in the CF. Strong absorption within the LP is anticipated, whereas within the TP $\hat{\eta}$ nearly coincides with $\hat{\eta}^{\mathrm{FP}}$.

Another way to understand this asymmetry is to follow photon trajectories in a reference frame (with primed quantities) where the condition

$\boldsymbol{E}^{\prime}=0$

is fulfilled. In such a frame, an asymmetry in $R^{\prime}$ (cf. Eq. (1)) for the LP and TP photons arises from transformation 

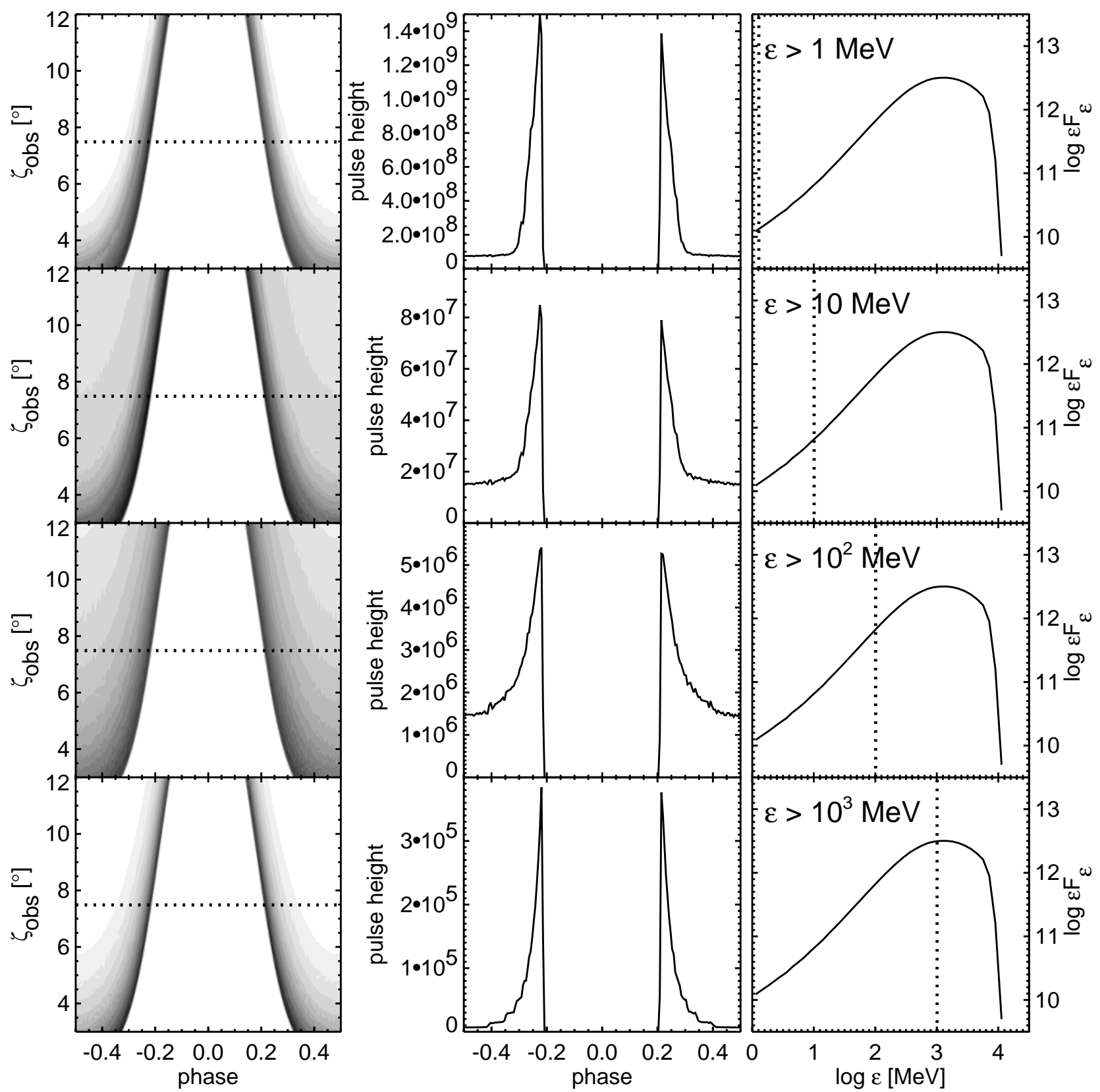

Fig. 3a. Directional and spectral gamma-ray characteristics calculated for the Vela pulsar with the angle $\alpha$ between the spin axis and the magnetic axis set $\alpha=7.6^{\circ}$ (nearly aligned rotator). Eight rows are shown (the continuation in Fig. 3b), with three panels each. Left column shows the outgoing photons of energy $\varepsilon>\varepsilon_{\text {limit }}$ which are mapped onto the parameter space $\zeta_{\text {obs }}$ vs. $\phi$, where $\zeta_{\text {obs }}$ is the viewing angle (between the spin axis and the 1.o.s) and $\phi$ denotes the phase of rotation. Middle column shows the double-peak pulse profile formed with these photons when $\zeta_{\text {obs }}=7.6^{\circ}$ is chosen (yielding the peak-to-peak separation equal 0.42). Right column shows the phase-averaged energy spectrum (the flux level $\varepsilon F_{\varepsilon}$ in arbitrary units) for $\zeta_{\text {obs }}=7.6^{\circ}$ i.e. the same for all rows. Dotted vertical line indicates the part of the spectrum $\left(\varepsilon>\varepsilon_{\text {limit }}\right)$ which contributes to the corresponding pulse profile on the left. The eight rows correspond to 8 consequtive values of $\varepsilon_{\text {limit }}: 1,10,10^{2}, 10^{3}, 4 \times 10^{3}$, $6 \times 10^{3}, 8 \times 10^{3}$, and $10^{4} \mathrm{MeV}$ (these values are displayed in the panels of the right column).

properties of $\theta_{B}^{\prime}$ (aberration), $\varepsilon^{\prime}$ (red- or blue-shift), and $B^{\prime}$. One of the reference frames satisfying the condition (2) is a reference frame of local $\boldsymbol{E} \times \boldsymbol{B}$ drift. Denoting dimensionless drift velocity $\boldsymbol{\beta}_{D}=\boldsymbol{E} \times \boldsymbol{B} / \boldsymbol{B}^{2}$, the transformations read:

$$
\begin{gathered}
\varepsilon^{\prime}=\varepsilon \gamma_{D}\left(1-\eta_{x} \beta_{D}\right), \quad \boldsymbol{B}^{\prime}=\frac{\boldsymbol{B}}{\gamma_{D}}, \\
\sin \theta_{B}^{\prime}=\frac{\left[\left(\eta_{x}-\beta_{D}\right)^{2}+\eta_{y}^{2}\left(1-\beta_{D}^{2}\right)\right]^{1 / 2}}{\left(1-\eta_{x} \beta_{D}\right)} .
\end{gathered}
$$

In the equatorial plane of orthogonal rotator $\eta_{y}=0$ so that $\eta_{x}=\mp \sin \theta_{B}$, where the signs "minus" and "plus" correspond to the leading and the trailing peak, respectively. The transformation of propagation angle reduces then to $\sin \theta_{B}^{\prime}=$ $\left|\eta_{x}-\beta_{D}\right| /\left(1-\eta_{x} \beta_{D}\right)$. The Taylor expansion of $\sin \theta_{B}^{\prime}, \varepsilon^{\prime}$, and $B^{\prime}$ around $\beta_{D}=0$ reveals that a difference between the magnetic absorption rates in the locally drifting frame and in the OF results primarily from the aberration of photon direction, whereas 

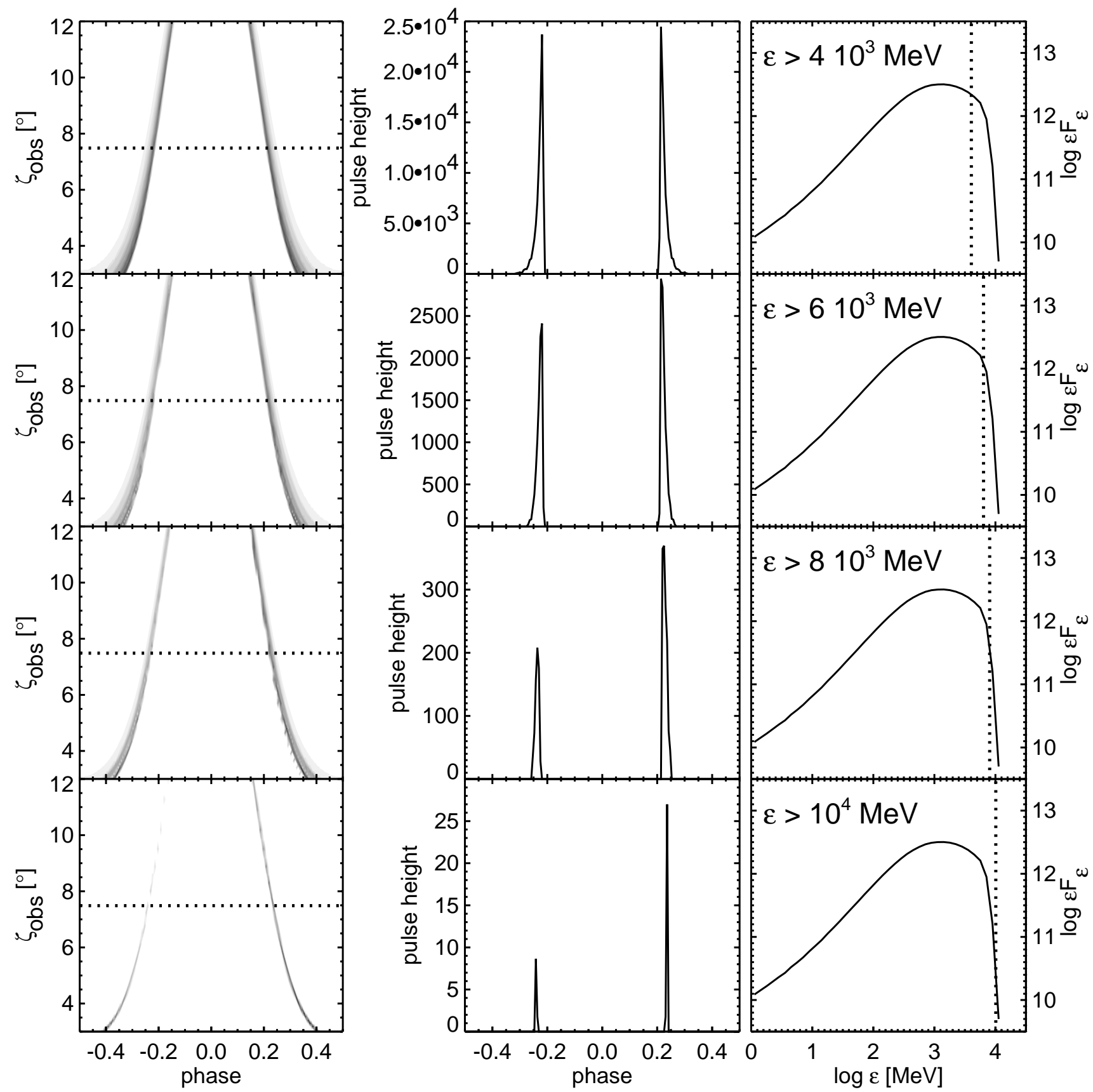

Fig. 3b. Continued.

the changes in $\varepsilon$ and $\boldsymbol{B}$ are second order effects. Obviously, the aberration is asymmetric for the LP and the TP $\left(\eta_{x}<0\right.$ and $\eta_{x}>0$, respectively). Figure 1 presents this "aberration effect" in the rigidly corotating frame, where $\boldsymbol{E}^{\prime}=0$ is assumed. Photon trajectories in this frame (dashed curves) indicate clearly that photons of the leading peak encounter larger $B_{\perp}^{\prime}$ than photons of the trailing peak of the same energy.

\section{Numerical modelling of the gamma-ray data}

We performed Monte Carlo simulations of curvature-radiationinduced electromagnetic cascades developing above a polar cap. The cascade development due to magnetic photon absorption accompanied by $e^{ \pm}$-pair creation and then synchrotron emission was followed in a $3 \mathrm{D}$ space in order to analyse pulse properties. As an example we choose a model with basic parameters of the Vela pulsar: $B_{\mathrm{pc}}=6.8 \mathrm{TG}$, $P=0.0893$ s. In order to meet observational restrictions for the Vela, both spectral and temporal, the following general requirements within polar-cap scenarios had to be satisfied: 1) a polar-cap accelerator should be placed a few stellar radii above pulsar's surface (Dyks et al. 2001); 2) an inclination angle $\alpha$ of the magnetic dipole with respect to the spin axis must not be large, and the pulsar has to be a nearly-aligned rotator (Daugherty \& Harding 1994). Recently Harding \& Muslimov (1998) proposed a physical mechanism for lifting the polar cap 


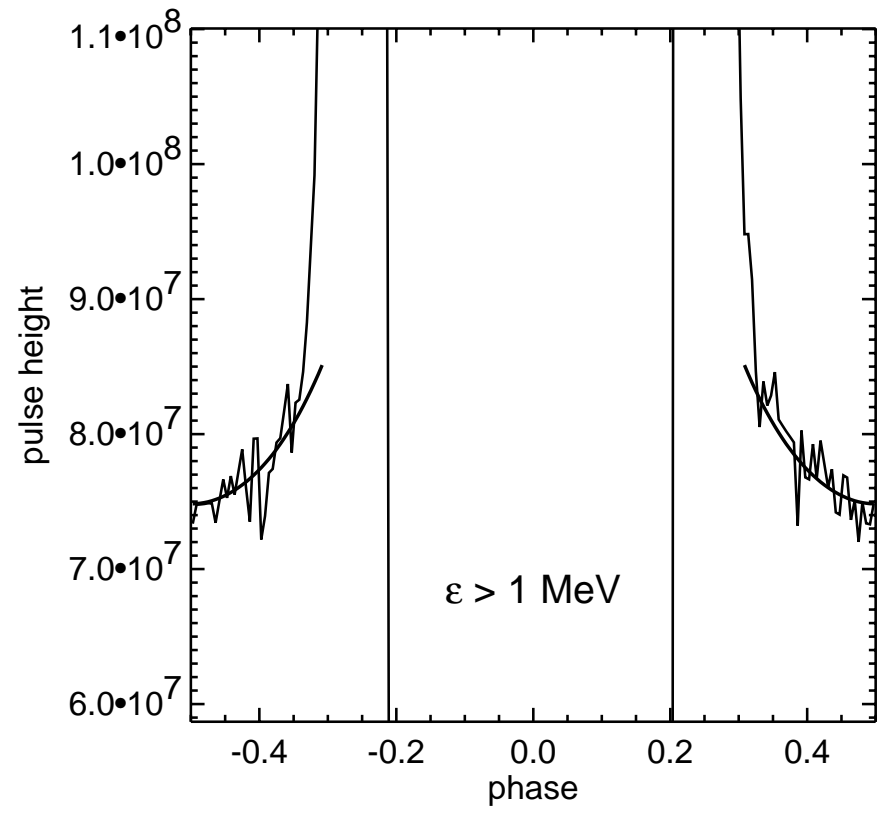

Fig. 4. Close-up view of the pulse profile for $\varepsilon>1 \mathrm{MeV}$ from Fig. 3a. The curvature-radiation wings $(|\phi|>0.3)$ accompanying both peaks can be now acknowledged. Their shape is modelled analytically (shown with thick solid lines).

accelerator up to $0.5-1 R_{\mathrm{NS}}$ above the surface. However, this altitude is still too low to explain the $10 \mathrm{GeV}$ radiation emerging the Vela magnetosphere unattenuated. Therefore, we placed the polar-cap accelerator at the altitude of $h_{0}=4 R_{\mathrm{NS}}$ to ensure that the magnetosphere is not entirely opaque to curvature photons of energy $\$ 10 \mathrm{GeV}$ (see Dyks et al. 2001 for the detailed model spectral fitting for the Vela pulsar). Similarly, Miyazaki \& Takahara (1997) achieved the best agreement between the observed and their modelled pulse profiles of the Crab pulsar placing the accelerator at $h_{0}=4 R_{\mathrm{ns}}$. To reproduce the observed peak-to-peak separation $\Delta^{\text {peak }} \simeq 0.42$ (Kanbach et al. 1994) we assumed (after Dyks \& Rudak 2000) for the inclination angle $\alpha$ and the observer's angle $\zeta_{\text {obs }}$ (an angle between the line-ofsight and the spin axis) that $\alpha=\zeta_{\text {obs }}=7.6^{\circ}$.

Our numerical results are presented in Fig. $3(a+b)$. The three columns of Fig. 3 show (from left to right): 1) mapping onto the parameter space $\zeta_{\text {obs }}$ vs. $\phi$ of outgoing photons with energy $\varepsilon>\varepsilon_{\text {limit }}$ (where $\phi$ denotes a phase of rotation), 2) double-peak pulse profile due to these photons when $\zeta_{\text {obs }}=7.6^{\circ}$, and 3) phase-integrated energy spectrum of these photons, with the position of $\varepsilon_{\text {limit }}$ indicated with dotted vertical line. The eight rows correspond to 8 different values of $\varepsilon_{\text {limit }}: 1,10,10^{2}, 10^{3}, 4 \times 10^{3}, 6 \times 10^{3}, 8 \times 10^{3}$, and $10^{4} \mathrm{MeV}$ (top to bottom). An asymmetry in the double-peak profiles is noticable even though the rotator is nearly aligned: at the highest energies, above $\sim 6 \mathrm{GeV}$, the leading peak LP is less intense than the trailing peak TP (three lowermost panels in the middle column in Fig. 3b). This is a direct result of stronger magnetic absorption of the LP photons comparing to the TP photons. The distribution of these photons in the corresponding panels of $\zeta_{\text {obs }}$ vs. $\phi$ (the left column) shows that at viewing angles $\zeta_{\text {obs }}$ larger than $7.6^{\circ}$ (not allowed due to the fixed peak-to-peak separation of 0.42 ) the asymmetry in pulse profile would be even stronger. This demonstrates an increasing role of rotational effects as the distance from the spin axis increases.

In the course of magnetic absorption high-energy curvature photons are converted into electron-positron pairs which in turn emit low-energy synchrotron photons. Asymmetry in the absorption rate as discussed above means, therefore, an identical asymmetry in the $e^{ \pm}$pair production rate. Consequently, higher number of low-energy synchrotron photons emerges at the LP than at the TP. This is the reason for a dominance of the LP over the TP below $\sim 100 \mathrm{MeV}$, noticable in Fig. 3a. Combining the results from both energy domains, a characteristic inversion in the relative strentgh of the LP and the TP occurs across the gamma-ray energy space. A qualitatively similar inversion of peak intensities takes place in the gamma-ray double-pulse of the Vela pulsar (Thompson 2001).

The beam of synchrotron radiation in our cascades occupies a very narrow range of magnetic colatitudes; in other words it is highly anisotropic. The reasons for this include a very limited range of altitudes at which the $e^{ \pm}$pairs are created and the effects of relativistic beaming. By comparison, curvature radiation below $\sim 100 \mathrm{MeV}$ is much less anisotropic. Therefore, the prominent peaks visible at $\varepsilon<100 \mathrm{MeV}$ (two uppermost panels of Fig. 3a) consist almost entirely of synchrotron radiation (SR) photons, whereas the apparently flat wings outside the peaks (i.e. within the "offpulse" region corresponding to high altitudes) are composed of curvature radiation (CR) photons. A close-up view of the double-peak pulse profile for $\varepsilon>1 \mathrm{MeV}$ shown in Fig. 4 reveals that the CR wings are not flat - in fact their intensity decreases with increasing phase $|\phi|$; moreover, their shapes can be reproduced with analytical means: spectral power of curvature radiation $\frac{\mathrm{d} P_{\mathrm{cr}}}{\mathrm{d} \varepsilon}$ well below a characteristic photon energy $\varepsilon_{\text {crit }} \propto \frac{\gamma^{3}}{\rho_{\text {cr }}}$ does not depend on the energy of radiating particles $\gamma$ but on the curvature radius $\rho_{\text {cr }}$ of magnetic field lines solely. Since primary electrons reside within a pulsar magnetosphere for a limited period of time $\varepsilon_{\text {crit }}$ has a lower limit which equals roughly $\lesssim 100 \mathrm{MeV}$ (see Rudak \& Dyks 1999 for details). Therefore, the wings in the pulse profiles below $100 \mathrm{MeV}$ fall off due exclusively to an increase in the curvature radius $\rho_{\mathrm{cr}}$ of magnetic field lines: this proceeds according to the following relation

$\frac{\mathrm{d} P_{\mathrm{cr}}}{\mathrm{d} \varepsilon} \propto \rho_{\mathrm{cr}}^{-2 / 3}$,

which then leads to the smooth solid lines in Fig. 4. As the photon energy increases and the strength of the synchrotron peaks decreases the curvature wings become more and more pronounced. They are most noticeable near $100 \mathrm{MeV}$. Above $100 \mathrm{MeV}$ the wings gradually disappear (see the middle column in Fig. 3b) because radiating electrons are not energetic enough at high altitudes. At the same time the peaks become narrower - an effect noticed by Kanbach et al. (1994) in the EGRET data for the Vela pulsar.

As noted by Daugherty \& Harding (1996) the wings within the offpulse region must not be too strong within the entire energy range of EGRET if the theoretical pulse profiles are to resemble those of the Vela pulsar. We find that the intensity of wings relative to the intensity of peaks depends sensitively 

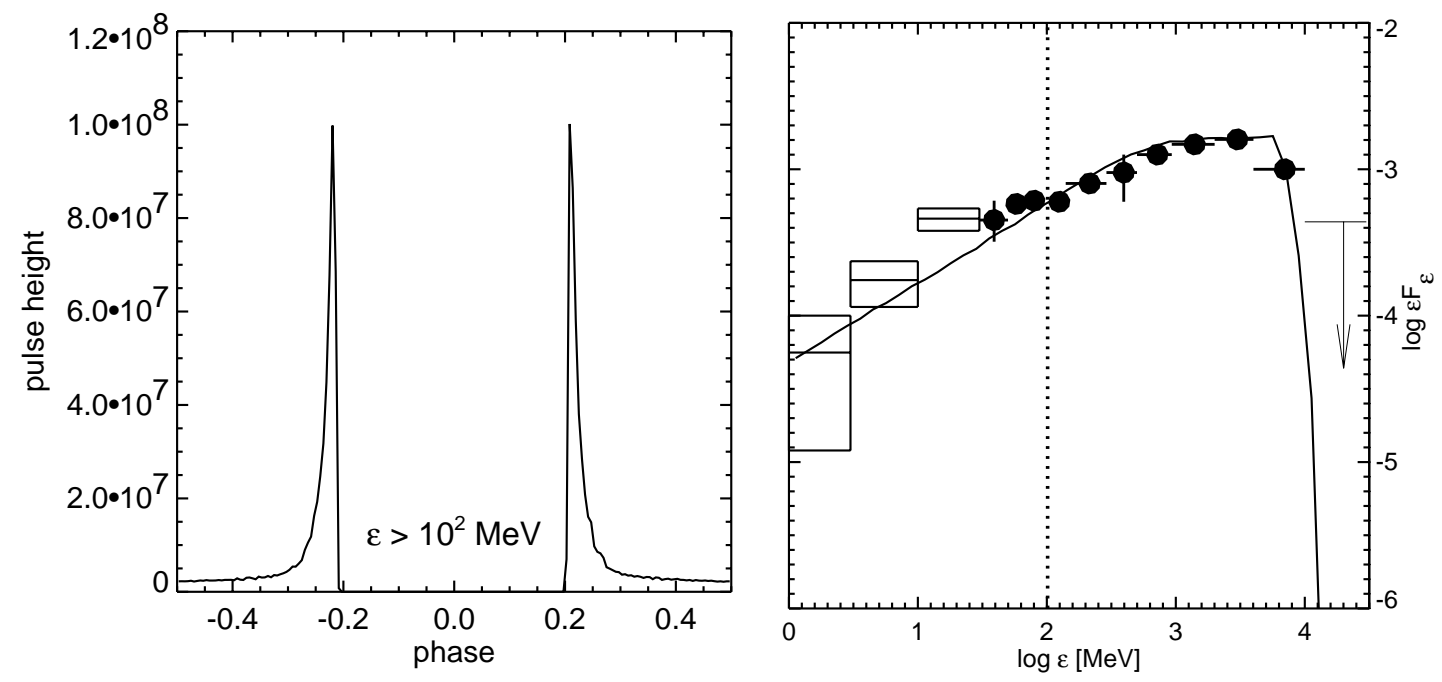

Fig. 5. Pulse profile integrated for $\varepsilon>100 \mathrm{MeV}$ (left panel) and the phase averaged energy spectrum (right panel) are shown for the case of initial energy of primary electrons $E_{0}=2 \times 10^{7} \mathrm{MeV}$. Note the relatively weaker wings outside the peaks and much softer spectrum in comparison with the previous case of $E_{0}=10^{7} \mathrm{MeV}$ (Fig. 3a, third row from the top). The data for the Vela pulsar are laid over the model spectrum: boxes are the data from the first COMPTEL source catalogue (see Table 3 in Schönfelder et al. 2000), and dots (plus an upper limit above $10 \mathrm{GeV}$ ) are the EGRET data (Thompson et al. 1997). The flux level $\varepsilon F_{\varepsilon}$ is in $\mathrm{MeV} \mathrm{cm}^{-2} \mathrm{~s}^{-1}$ units.

on the richness of the cascades, i.e. on the multiplicity $\mathcal{M}$ (the number of created pairs per primary electron). The results discussed above and presented in Fig. 3 had been obtained for the initial energy of primary electrons $E_{0}=10^{7} \mathrm{MeV}$ which yielded $\mathcal{M}=73$. By increasing the initial energy $E_{0}$ up to $2 \times 10^{7} \mathrm{MeV}$ the multiplicity reaches $\mathcal{M}=830$ and the corresponding pulse profile at $100 \mathrm{MeV}$ (left panel of Fig. 5) changes notably with respect to its counterpart of Fig. 3a. It reveals now a much lower level of wings outside the peaks. Equally important is the change in the shape of the phaseaveraged energy spectrum which becomes much softer by gaining more power in the low-energy range (right panel in Fig. 5). Both new features are in rough agreement with the data for the Vela pulsar, contrary to the case with $E_{0}=10^{7} \mathrm{MeV}$. It is interesting to note that the association of the broad peaks at $100 \mathrm{MeV}$ with the relatively hard spectrum (Fig. 3a) on one hand, and of the narrow peaks with the soft spectrum (Fig. 5) on the other hand do resemble qualitatively the observed characteristics of Geminga and the Vela pulsar, respectively.

We may now test our model of the double-peak asymmetry by comparing the numerical results obtained for specific pulsar parameters with the data for real objects. Since the effect is induced by magnetic absorption the expected weakening of the leading peak with respect to the trailing peak occurs only in the vicinity of the high-energy spectral cutoff. Therefore, it is essential to have good photon statistics also at the highest energy bins, i.e. above $1 \mathrm{GeV}$. As far as the EGRET data are concerned this requirement is barely satisfied even for Vela. With these limitation in mind, we consider Vela as the only appropriate case to provide the test. We used the EGRET data for Vela to calculate the ratio (denoted as $P 2 / P 1$ ) of the photon counts in the LP and the TP (denoted as $P 1$ and $P 2$, respectively). For each energy bin (the energy bins cover the range between $\sim 30 \mathrm{MeV}$ and $\sim 10 \mathrm{GeV}$ ) we calculated $P 1(P 2)$ by summing all photons within the range $\phi_{\mathrm{lp}} \pm 0.05\left(\phi_{\mathrm{tp}} \pm 0.05\right)$ in phase, where $\phi_{\mathrm{lp}}\left(\phi_{\mathrm{tp}}\right)$ is the phase of maximum in the LP (the TP) at $100 \mathrm{MeV}$. Figure 6 shows the observational points as well as their estimated errors ${ }^{1}$ along with the results of model calculations performed for three different altitudes: $h_{0}=2 R_{\mathrm{NS}}, 3 R_{\mathrm{NS}}$, and $4 R_{\mathrm{NS}}$. The overall qualitative and quantitative behaviour of $P 2 / P 1$ for the EGRET data is very similar to the dependence presented by Kanbach et al. 1980 for the COS-B data. The data points certainly can acommodate our model. However, to answer the question of whether it would be necessary to invoke any additional processes to reproduce the increase in $P 2 / P 1$ inferred from the data requires better photon statistics at the spectral cut-off and careful statistical analysis.

\section{Rotational asymmetry as a function of pulsar parameters}

The asymmetry effects are marginal for nearly aligned pulsars with periods $P \sim 0.1 \mathrm{~s}$. For the above-described model of Vela, they are noticeable only because of the high altitude of the accelerator $\left(h_{0}=4 R_{\mathrm{NS}}\right)$. However, for highly inclined $\left(\alpha \gtrsim 45^{\circ}\right)$ and fast pulsars $(P \lesssim 10 \mathrm{~ms})$ the rotational effects result in asymmetry of considerable magnitude. If detected by GLAST, $\mathrm{GeV}$ emission from such objects would provide powerful diagnostics of the polar cap model. Below we present the magnitude of such asymmetry predicted for a wide range of parameters for fast pulsars.

As a measure of rotational effects we consider the escape energy $\varepsilon_{\text {esc }}$ which is defined as a maximum energy of a photon (and the photon is emitted tangentially to its "parent" magnetic field line; a footpoint of this line has magnetic colatitude $\theta$ ) for which the magnetosphere is still transparent, i.e. for which the optical depth integrated along the photon trajectory is less

${ }^{1}$ Detailed analysis of the data will be presented elsewhere (Woźna et al. 2002, in preparation). 


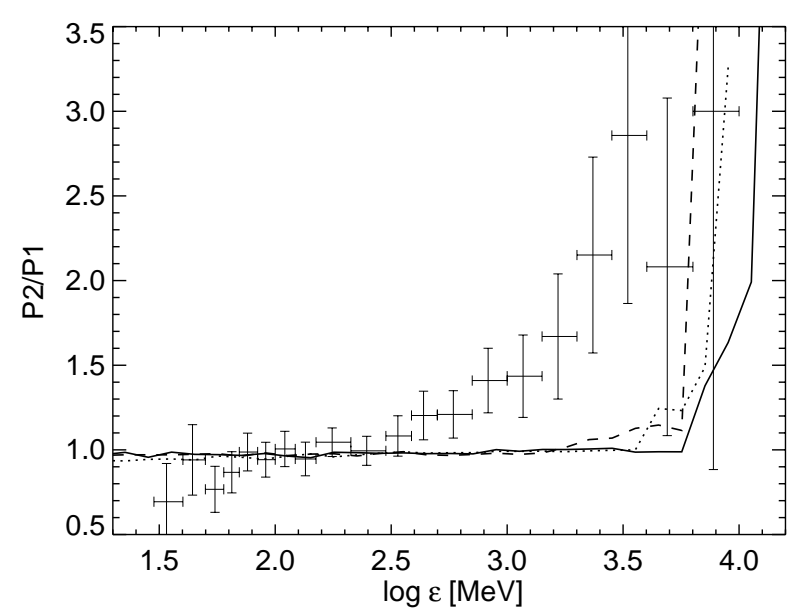

Fig. 6. The ratio of the number of photons in the trailing peak and the leading peak $P 2 / P 1$ inferred from the EGRET data for Vela is shown in function of photon energy $\varepsilon$. The results of model calculations performed for three different values of the altitude $h_{0}$ of the accelerator are indicated with three lines: dashed, dotted, and solid for $h_{0}=2 R_{\mathrm{NS}}, 3 R_{\mathrm{NS}}$, and $4 R_{\mathrm{NS}}$, respectively.

than 1. This energy was calculated with our numerical code and the results are shown below. For the sake of comparison with the case of no rotational effects we recall a simplified, yet quite accurate ${ }^{2}$ for magnetic fields weaker than $\sim 0.1 B_{\mathrm{cr}}$, analytic formula which (after e.g. Bulik et al. 2000) reads

$\varepsilon_{\mathrm{esc}}(\theta)=\frac{\theta_{\mathrm{pc}}}{\theta} \cdot \varepsilon_{\mathrm{esc}}\left(\theta_{\mathrm{pc}}\right)$,

where

$\varepsilon_{\mathrm{esc}}\left(\theta_{\mathrm{pc}}\right) \approx 1 \mathrm{GeV}\left(\frac{B_{\mathrm{pc}}}{0.1 B_{\mathrm{cr}}}\right)^{-1}\left(\frac{\theta_{\mathrm{pc}}}{0.01 \mathrm{rad}}\right)^{-1}\left(\frac{r_{\mathrm{m}}}{R_{\mathrm{NS}}}\right)^{5 / 2}$.

Here $r_{\mathrm{m}}$ is the radial coordinate of the emission point (with spherical coordinates $\left(r_{\mathrm{m}}, \theta_{\mathrm{m}}, \phi_{\mathrm{m}}\right)$ in the right-handed frame with $\hat{z}$-axis along the dipole axis) and $\theta \ll 1 \mathrm{rad}$ is the magnetic colatitude of a footpoint of the parent magnetic field line at the neutron star surface. For field lines originating at the polar-cap rim one should take then $\theta=\theta_{\mathrm{pc}}$, where $\theta_{\mathrm{pc}}=$ $\left.\arcsin \left(\sqrt{(} R_{\mathrm{NS}} / R_{\mathrm{lc}}\right)\right) \approx 1.45 \times 10^{-2} / \sqrt{P}$ radians, and $R_{\mathrm{lc}}=c P / 2 \pi$ is the light cylinder. Note that the escape energy of Eq. (5) is symmetrical with respect to the dipole axis and goes to infinity at the magnetic pole $(\theta=0)$. Such a behaviour is not the case, however, when the rotational effects discussed in Sect. 3 are taken into account.

In Figs. 7, 8, and 9 we present the values of $\varepsilon_{\text {esc }}$ obtained numerically for fast rotators with $B_{\mathrm{pc}}=1 \mathrm{TG}$, and emission points located on the polar cap surface, i.e. $r_{\mathrm{em}}=R_{\mathrm{NS}}$ was assumed everywhere. Let us begin with the case of orthogonal rotators (i.e. $\alpha=90^{\circ}$ ) - these are shown in Figs. 7 and 8. Here we consider emission points lying along the crossection of polar cap surface with the equatorial plane of rotation (hence, the results would be relevant for observers located at $\zeta_{\text {obs }}=90^{\circ}$ ). Figure 7 shows how $\varepsilon_{\text {esc }}$ varies with location of the emission

\footnotetext{
${ }^{2}$ For presentation of detailed numerical results see e.g. Harding et al. (1997).
}

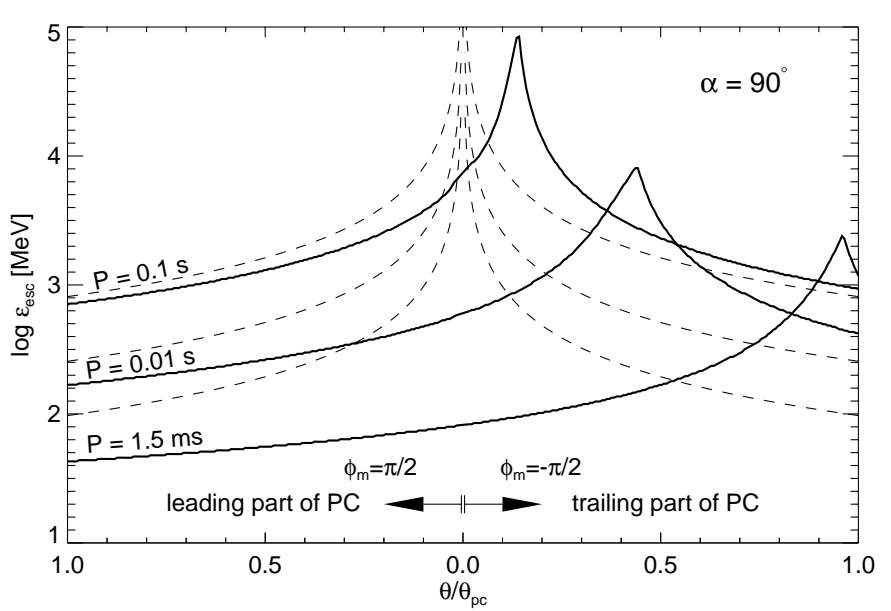

Fig. 7. The escape energy $\varepsilon_{\text {esc }}$ of photons from the polar cap surface of an orthogonal rotator with $B_{\mathrm{pc}}=10^{12} \mathrm{G}$ is shown as a function of normalized magnetic colatitude $\theta / \theta_{\mathrm{pc}}$ of the emission points. The points are assumed to lay along the cross-section of the polar cap surface with the equatorial plane of rotation, thus location of each point is determined by $\theta / \theta_{\mathrm{pc}}$ in the range $[0,1]$, and the magnetic azimuth $\phi_{\mathrm{m}}$ equal either to $\pi / 2$ (for the leading half of the polar cap) or $-\pi / 2$ (for the trailing half). Three solid lines are labelled with the corresponding spin periods $P$ of $0.1 \mathrm{~s}, 10 \mathrm{~ms}$, and $1.5 \mathrm{~ms}$. Moreover, to illustrate the difference with the case when rotational effects are ignored, each solid line is accompanied by a dashed line drawn according to Eq. (5).

point across the polar cap. The location of each point is determined by the normalized magnetic colatitude $\theta / \theta_{\mathrm{pc}}$ in the range $[0,1]$ and the magnetic azimuth $\phi_{\mathrm{m}}$ equal either to $\pi / 2$ (for the leading half of the polar cap) or $-\pi / 2$ (for the trailing half). The spin periods of $0.1 \mathrm{~s}, 10 \mathrm{~ms}$, and $1.5 \mathrm{~ms}$ were considered.

In order to quantify the asymmetry in $\varepsilon_{\text {esc }}$ between the leading and the trailing parts of the polar cap we introduce the following parameter:

$\mathcal{R}_{\mathrm{esc}}(\theta)=\frac{\varepsilon_{\mathrm{esc}}^{\mathrm{tp}}}{\varepsilon_{\mathrm{esc}}^{\mathrm{lp}}}$,

where

$\varepsilon_{\mathrm{esc}}^{\mathrm{lp}} \equiv \varepsilon_{\mathrm{esc}}\left(\phi_{\mathrm{m}}=\pi / 2, \theta\right)$,

$\varepsilon_{\mathrm{esc}}^{\mathrm{tp}} \equiv \varepsilon_{\mathrm{esc}}\left(\phi_{\mathrm{m}}=-\pi / 2, \theta\right)$.

For example, $\mathcal{R}_{\mathrm{esc}}\left(\theta_{\mathrm{pc}}\right)$ gives the asymmetry between two opposite points located on the outer rim of the polar cap. For spin periods around $0.1 \mathrm{~s}$, typical for the known gamma-ray pulsars, $\mathcal{R}_{\mathrm{esc}}\left(\theta_{\mathrm{pc}}\right)$ remains close to unity; e.g. for the case of $P=0.1 \mathrm{~s}$ in Fig. $7, \mathcal{R}_{\mathrm{esc}}\left(\theta_{\mathrm{pc}}\right) \simeq 1.3$. However, for $P$ smaller than $0.01 \mathrm{~s}$ the parameter $\mathcal{R}_{\mathrm{esc}}\left(\theta_{\mathrm{pc}}\right)$ becomes definitely larger: $\sim 2.5$ for $P=0.01 \mathrm{~s}$ and $\sim 30$ for $P=1.5 \mathrm{~ms}$. Thus, for pulsars with $P \lesssim 0.01 \mathrm{~s}$ and large inclination angles $\alpha$ we predict a notable difference (in excess of half a decade in photon energy) between the positions of high-energy spectral cutoff in the gamma-ray emission from the leading and the trailing part of the polar cap.

Another interesting implication of fast rotation is that $\varepsilon_{\text {esc }}$ has finite values for any $\theta$, including $\theta=0$ (the magnetic pole), in contrast to the "static" case of Eq. (5). This can be understood in the following way: consider emission points with 
decreasing colatitude $\theta$ in the leading part of the polar cap. As we approach the dipole axis $(\theta \rightarrow 0), \varepsilon_{\text {esc }}$ increases because the decreasing curvature of magnetic field lines leads to smaller angles between $\boldsymbol{B}^{\prime}$ and the photon propagation direction $\hat{\eta}^{\prime}$ in the corotating frame CF. However, photon trajectory bends backwards in the CF (see dashed lines in Fig. 1), which implies that also photons emitted at $\theta=0$ along the straight dipolar axis will quickly encounter $B_{\perp}^{\prime} \neq 0$, thus being subject to the magnetic absorption. Now entering the trailing part of the polar cap leads to further increase of $\varepsilon_{\text {esc }}$. This is because magnetic field lines start to bend in the same direction as the photon trajectory in the CF (in other words - the efficiency of absorption decreases for emission points in the trailing part of the polar cap). Eventually, at some point (we denote it as $\theta_{0}$ ) the escape energy reaches a maximum. This is the point where the magnetic field slippage along with the aberration of photon direction ensure small angles between $\boldsymbol{B}^{\prime}$ and $\hat{\eta}^{\prime}$ over large distances in the photon trajectory. Therefore, the faster is the rotation, the larger is the colatitude $\theta_{0}$ of that point. For example, $\theta_{0} / \theta_{\mathrm{pc}} \simeq 0.15$ for $P=0.1 \mathrm{~s}$ whereas $\theta_{0} / \theta_{\mathrm{pc}} \simeq 0.42$ for $P=0.01 \mathrm{~s}$ (see Fig. 7). For $P=1.5 \mathrm{~ms}$ this maximum occurs ${ }^{3}$ close to the outer rim (in the trailing part) and therefore huge asymmetry with respect to the leading rim is predicted: $\mathcal{R}_{\mathrm{esc}}\left(\theta_{\mathrm{pc}}\right) \simeq 30$. With further increase of $\theta$ (towards the trailing rim of the polar cap) local magnetic field lines start to bend stronger than the photon trajectory in the $\mathrm{CF}$, and this is why $\varepsilon_{\text {esc }}$ should now decrease. However, this decrease never compensates the asymmetry in $\varepsilon_{\mathrm{esc}}$ at $\theta_{\mathrm{pc}}$ with respect to $\theta=0$, i.e. one always ends up with $\mathcal{R}_{\mathrm{esc}}\left(\theta_{\mathrm{pc}}\right)>1$.

Figure 8 presents escape energy $\varepsilon_{\mathrm{esc}}^{\mathrm{lp}}$ and $\varepsilon_{\mathrm{esc}}^{\mathrm{tp}}$ as a function of spin period $P$. This energy was calculated for a fixed position $\theta_{\mathrm{fxd}}$ of the emission point, in order to highlight its dependence on rotation. We chose three pairs of oppositely located emission points at: $\theta_{\mathrm{fxd}}=2.6,8.3$, and $22^{\circ}$, which corespond to $\theta_{\mathrm{pc}}$ for $P=0.1 \mathrm{~s}, 10 \mathrm{~ms}$, and $1.5 \mathrm{~ms}$, respectively. As in Fig. 7, the emission points were placed at the neutron star surface, in the equatorial plane of the orthogonal rotator. In the case of slow rotation $(P \sim 1 \mathrm{~s})$, the values of $\varepsilon_{\mathrm{esc}}^{\mathrm{lp}}\left(\theta_{\mathrm{fxd}}\right)$ for the leading point and $\varepsilon_{\mathrm{esc}}^{\mathrm{tp}}\left(\theta_{\mathrm{fxd}}\right)$ for the trailing point are practically identical, and well approximated by Eq. (5). As rotation becomes faster ( $P$ around $\sim 0.1 \mathrm{~s}) \varepsilon_{\mathrm{esc}}^{\mathrm{lp}}\left(\theta_{\mathrm{fxd}}\right)$ and $\varepsilon_{\mathrm{esc}}^{\mathrm{tp}}\left(\theta_{\mathrm{fxd}}\right)$ start to diverge due to the asymmetric influence of $\boldsymbol{E}$. At even shorter periods, below

${ }^{3}$ It is easy to reproduce the behaviour of $\theta_{0} / \theta_{\mathrm{pc}}$ as a function of $P$ in Fig. 7 with analytic formula: the maximal value of $B_{\perp}(r) / B_{\mathrm{pc}}$ encountered in a static dipolar field by a photon emitted along the local field line at $\theta_{0}$ is approximately equal to $0.1 \theta_{0}$ (Sturrock 1971, also Fig. 1 in Rudak \& Ritter 1994). This occurs always at $r_{0}=4 / 3 R_{\mathrm{NS}}$, regardless the value of $\theta_{0}$. The angle $\psi$ between the photon propagation direction and the local field line at $r_{0}$ is $\psi_{0} \approx B_{\perp}\left(r_{0}\right) / B\left(r_{0}\right)$ and therefore $\psi_{0} \approx 0.1 \theta_{0}(4 / 3)^{3}$. To minimize $B_{\perp}^{\prime}\left(r_{0}\right)$ as much as possible in the case of rotation, the aberration angle due to local linear velocity $\beta\left(r_{0}\right)=4 / 3 R_{\mathrm{NS}} / R_{\mathrm{lc}}$ should be close to $\psi_{0}$. Since $\beta\left(r_{0}\right) \ll 1$, the aberration angle is $\sim \beta\left(r_{0}\right)$. Therefore, we obtain the condition $0.1(4 / 3)^{2} \theta_{0} \approx \theta_{\mathrm{pc}}^{2}$ which gives

$$
\frac{\theta_{0}}{\theta_{\mathrm{pc}}} \approx 0.08 P^{-1 / 2} \text {. }
$$

This formula overestimates the locations of maxima in Fig. 7 by a factor $\sim 2$ only.

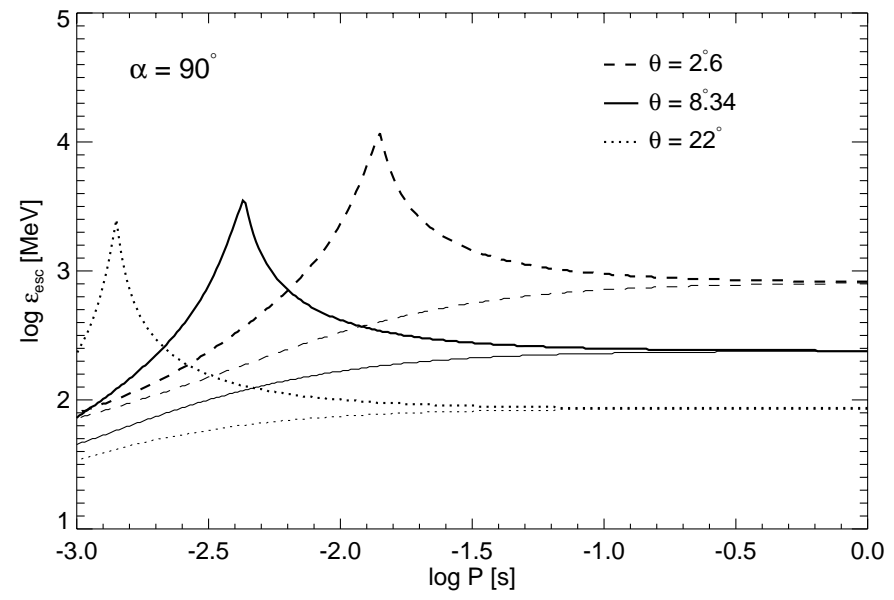

Fig. 8. Escape energy at fixed magnetic colatitude $\theta$ as a function of rotation period. The dashed, solid, and dotted lines correspond to $\theta=$ $2.6^{\circ}, 8.3^{\circ}$, and $22^{\circ}$, respectively. The thin lines correspond to points on the leading side of the dipole axis (with spherical coordinates $\left(R_{\mathrm{NS}}\right.$, $\pi / 2, \theta)$ ). The thick lines correspond to points on the trailing side (with $\left.\left(R_{\mathrm{NS}},-\pi / 2, \theta\right)\right)$. The curves are for emission in the equatorial plane of orthogonal rotator.

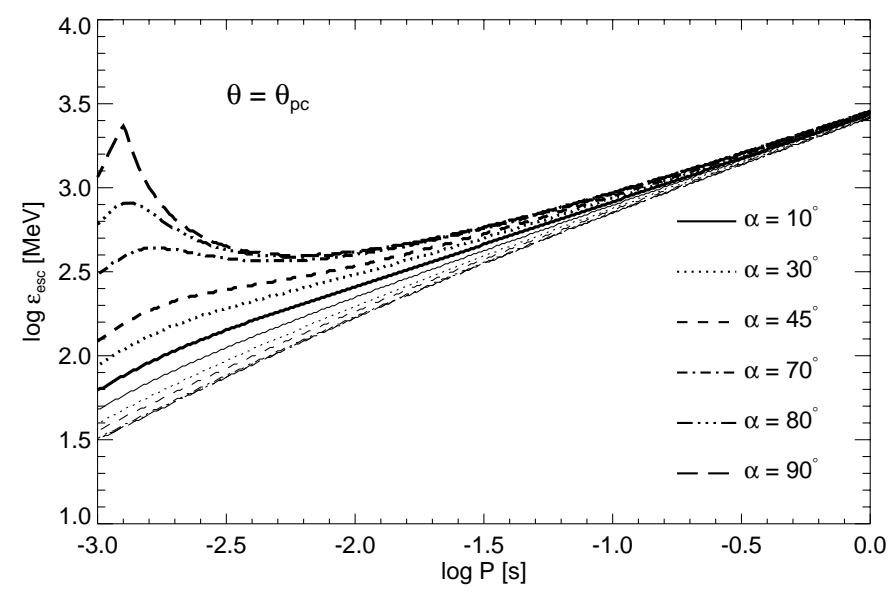

Fig. 9. Escape energy for the leading and the trailing emission points at the polar cap rim as a function of rotation period. Six uppermost lines (thick) correspond to the trailing points. Thin lines are for the leading points (the lines for $\alpha=70^{\circ}, 80^{\circ}$, and $90^{\circ}$ overlap).

$\sim 0.03 \mathrm{~s}$, the maxima in $\varepsilon_{\mathrm{esc}}^{\mathrm{tp}}$ are reached (at the values of $P$, which can be reproduced by solving Eq. (9) with $\theta_{\mathrm{fxd}}$ in place of $\theta_{0}$ ) because slippage of field lines starts to be important (see previous paragraph). For increasing $\theta_{\mathrm{fxd}}\left(2^{\circ} .6,8.3\right.$, and $22^{\circ}$ in Fig. 8), the asymmetry parameter $\mathcal{R}_{\text {esc }}\left(\theta_{\mathrm{fxd}}\right)$ decreases for slow rotators $(P \gtrsim 0.1 \mathrm{~s})$, and it increases for fast (millisecond) rotators.

In Fig. 9 we present $\varepsilon_{\mathrm{esc}}^{\mathrm{lp}}\left(\theta_{\mathrm{pc}}\right)$ and $\varepsilon_{\mathrm{esc}}^{\mathrm{tp}}\left(\theta_{\mathrm{pc}}\right)$ as a function of spin period $P$ for a set of dipole inclinations $\alpha$. Note that unlike in Fig. 8, the emission points are now placed at $\theta=\theta_{\mathrm{pc}}$, i.e. at the rim of polar cap corresponding to $P$. In the case of small inclinations (e.g. $\alpha=10^{\circ}$ ), the resulting ratio $\mathcal{R}_{\mathrm{esc}}\left(\theta_{\mathrm{pc}}\right)$ remains close to unity even for millisecond periods. The general increase in $\varepsilon_{\text {esc }}$ with $P$ increasing, noticeable in Fig. 9, reflects the approach of emission points to the dipole axis, where the curvature of magnetic field lines is small. The trend is well described 


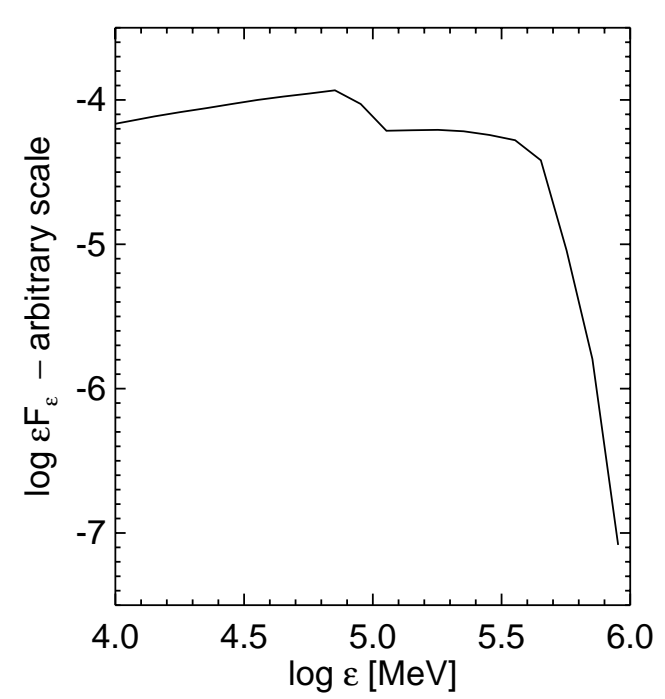

Fig. 10. Theoretical "on-beam" spectrum of high-energy emission from a millisecond pulsar with $P=2.3 \mathrm{~ms}, B_{\mathrm{pc}}=10^{9} \mathrm{G}$, and $\zeta_{\text {obs }}=\alpha=60^{\circ}$. The density distribution of primary electrons over the polar cap, which was assumed in this calculation, had a maximum at the polar cap rim and was uniform in magnetic azimuth $\phi_{\mathrm{m}}$. The magnetic colatitude profile of this distribution had a Gaussian shape centered at $\theta=\theta_{\mathrm{pc}}$, with a width $\sigma=0.05 \theta_{\mathrm{pc}}$. The initial energy of primary electrons was equal to $2 \times 10^{7} \mathrm{MeV}$. Note the step-like decline near $\sim 100 \mathrm{GeV}$.

by $\varepsilon_{\text {esc }} \propto \sqrt{P}$ as given by Eq. (6). In the range of spin periods below $\sim 0.1 \mathrm{~s}$ and with $\alpha \gtrsim 45^{\circ}$, the difference between $\varepsilon_{\mathrm{esc}}^{\mathrm{lp}}$ and $\varepsilon_{\text {esc }}^{\text {tp }}$ becomes pronounced, especially for highly inclined millisecond pulsars. If detected by GLAST, high-energy emission from such objects would provide an ideal test of the polar cap model.

However, for the asymmetry effects to be detectable, an additional condition (apart from short period and large inclination) must be fulfilled: the viewing geometry must be of the "on-beam" type, i.e. the observer's line of sight must cross the narrow beam of radiation at the high-energy cutoff - where the magnetic absorption operates. If this is the case, the asymmetry in the absorption may be noticeable even in the phaseaveraged spectra. As an example, we show in Fig. 10 the phaseaveraged spectrum calculated for a millisecond pulsar with $P=2.3 \mathrm{~ms}, B_{\mathrm{pc}}=10^{9} \mathrm{G}, \alpha=60^{\circ}$, and for $\zeta_{\mathrm{obs}}=60^{\circ}$. For this rotator we obtain $\varepsilon_{\mathrm{esc}}^{\mathrm{lp}} \simeq 10^{5} \mathrm{MeV}$ and $\varepsilon_{\mathrm{esc}}^{\mathrm{tp}} \simeq 5 \times 10^{5} \mathrm{MeV}$ at the rim of the polar cap. As a result of this rotationally induced asymmetry in magnetic absorption for the leading and the trailing peak the spectrum at its high-energy cutoff assumes a step-like shape: below $\simeq 10^{5} \mathrm{MeV}$ the spectrum consists of photons from both the leading and the trailing peak, whereas between $\sim 10^{5} \mathrm{MeV}$ and $\sim 5 \times 10^{5} \mathrm{MeV}$ only photons of the trailing peak contribute to the spectrum; at $\varepsilon \simeq \varepsilon_{\mathrm{esc}}^{\text {lp }}$ the level of the spectrum drops by a factor of $\sim 2$. In these particular calculations of the spectrum, we assumed that the density distribution of primary electrons over the polar cap is dominated by an outer-rim component (see the captions to Fig. 10 for details of the distribution).

If, however, an emission from the inner part of the polar cap were to contribute considerably to the outer-rim emission, the step-like shape shown in Fig. 10 would be smoothed out, because of contribution of many spectra with different values of $\varepsilon_{\text {esc }}$. In such a case the polar cap origin of the observed radiation could be easily revealed by noting strong differences between the high-energy spectral cutoffs in different ranges of the rotational phase (i.e. phase-resolved spectra would have to be obtained). The first obvious candidate to check for this effect (e.g. with GLAST) seems to be J0218+4232 - the only gamma-ray pulsar among all millisecond pulsars (Kuiper et al. 2000). However, this pulsar appears to be a candidate for an "off-beam" case (see Dyks \& Rudak 2002 for details).

The strength of magnetic field $B_{\mathrm{pc}}$ practically does not affect the shapes of curves shown in Figs. 7-9. The magnetic field only acts as a scaling factor: $\varepsilon_{\text {esc }} \propto B^{-1}$; cf. Eq. (6).

\section{Discussion}

We have shown that pulsar rotation induces an asymmetry in the magnetic absorption rate with respect to the magnetic dipole axis. Its consequences are potentially interesting in constraining the phase-space of parameters in the polar cap models of high-energy radiation, provided that very high quality gamma-ray data (e.g. as expected from GLAST) are at hand. Its magnitude depends mainly on the linear velocity $\beta$ of the magnetosphere at sites of particle acceleration and magnetic photon absorption. When the region of electron acceleration is placed just above the neutron star surface rotation does not produce any detectable effects even for relatively fast rotating young gamma-ray pulsars. However, it has been argued that at least in the case of the Vela pulsar, such a situation is difficult to reconcile with the spectral high-energy cutoff at about $10 \mathrm{GeV}$ (e.g. Dyks et al. 2001). We find then that raising the accelerator up to $\sim 4$ neutron star radii (in the spirit of Harding \& Muslimov 1998) above its polar cap produces asymmetric gamma-ray pulse profiles even in the case of nearly aligned rotators with a spin period of $P \sim 0.1 \mathrm{~s}$. The resulting features - softer spectrum of the leading peak and the dominance of the trailing peak above $\sim 5 \mathrm{GeV}$ - do agree qualitatively with the EGRET data of the bright gamma-ray pulsars (Thompson 2001).

We are far from concluding that the rotation effects alone can account for the observed asymmetry in the double peaks of the bright EGRET pulsars. On the contrary - some axial asymmetry intrinsic to the region of electron acceleration is inevitable in order to explain the double-peak properties at $100 \mathrm{MeV}$ of Geminga and B1706-44, where the leading peak is weaker than the trailing peak. Strong deviations of the actual magnetic field structure from the pure dipole at the stellar surface (e.g. Gil et al. 2002) might be responsible for maintaining axial asymmetry at the site of electron acceleration (unlike the symmetric initial conditions introduced in Sect. 2). This in turn would lead to electromagnetic cascades whose properties vary with magnetic azimuth. It is important, however, that the propagation effects due to rotation work in the right direction, i.e. they explain qualitatively the observed weakening of the leading peak with respect to the trailing peak. We emphasize that this weakening occurs only in the vicinity of the (phaseaveraged) high-energy spectral cutoff, where the flux level decreases significantly. 
Another consequence of the magnetic absorption of high energy photons is a noticeable change in the separation $\Delta^{\text {peak }}$ between the two peaks in the pulse, taking place near the highenergy spectral cutoff (Dyks \& Rudak 2000). In the model discussed above, with electrons ejected only from a rim of the polar cap, the higher energy of photons requires higher emission altitudes to avoid absorption. Therefore, a slight increase in $\Delta^{\text {peak }}$ is visible in the three lowermost pulse profiles in Fig. 3b. However, if the emission from the interior of the polar cap were included, just the opposite behaviour would occur: $\Delta^{\text {peak }}$ would decrease near the high-energy cutoff in the spectrum. This is because in this case of a "filled polar cap tube", the highest energy non-absorbed photons are emitted closer to the magnetic dipole axis (see Fig. 2 in Dyks \& Rudak 2000). The latter case agrees qualitatively with the marginal decrease in peak separation found in the EGRET data for Vela (Kanbach 1999).

Stimulated by high-quality observations of gamma-ray pulsars anticipated with GLAST we analysed in Sect. 5 the importance of rotation-driven asymmetry in magnetic absorption for a broad range of pulsar parameters. A decline in gamma-ray flux at high-energy spectral cutoff should inevitably be accompanied by strong changes in pulse profiles: whereas at lower photon energies the profile is determined by the density distribution of primary electrons over the polar cap and the efficiency of photon emission mechanism, in the vicinity of the cutoff it becomes additionally constrained by likely high values of the asymmetry parameter $\mathcal{R}_{\mathrm{esc}}\left(\theta_{\mathrm{pc}}\right)$ - the situation anticipated for fast rotating $(P<0.01 \mathrm{~s})$, and highly inclined $\left(\alpha \gtrsim 45^{\circ}\right)$ pulsars.

Acknowledgements. We thank V.S. Beskin and A.K. Harding for useful comments on the issue of magnetospheric distortions. We are grateful to Gottfried Kanbach for providing us with the EGRET data on Vela, and to Aga Woźna for calculating the P2/P1 ratios used in Fig. 6. We acknowledge comments and stimulating suggestions made by the anonymous referee. JD appreciates Young Researcher Scholarship of Foundation for Polish Science. This work was supported by KBN (grants 2P03D02117 and 5P03D02420) and NCU (grant 405A).

\section{References}

Baring, M. G. 2001, Polar cap pulsar models, in Proc. Tonantzintla Workshop, ed. A. Carramiñana, O. Reimer, \& D. J. Thompson, Astrophysics and Space Science Library, 267, 167

Baring, M. G., \& Harding, A. K. 2000, Bull. AAS, 32, 12.43
Beskin, V. S. 1999, Physics-Uspekhi, 42, 1071

Blaskiewicz, M., Cordes, J. M., \& Wasserman, I. 1991, ApJ, 370, 643

Bulik, T., Rudak, B., \& Dyks, J. 2000, MNRAS, 317, 97

Daugherty, J. K., \& Harding, A. K. 1982, ApJ, 252, 337

Daugherty, J. K., \& Harding, A. K. 1983, ApJ, 273, 761

Daugherty, J. K., \& Harding, A. K. 1994, ApJ, 429, 325

Daugherty, J. K., \& Harding, A. K. 1996, ApJ, 458, 278

Daugherty, J. K., \& Lerche, I. 1975, Ap\&SS, 38, 437

Dyks, J., \& Rudak, B. 2000, MNRAS, 319, 477

Dyks, J., \& Rudak, B. 2002, The spectrum of the millisecond pulsar J0218+4232 - theoretical interpretations, in Proc. of the XXII Moriond Meeting, in press [astro-ph/0205222]

Dyks, J., Rudak, B., \& Bulik, T. 2001, Model spectra of rotation powered pulsars in the INTEGRAL range, in Proc. of the 4th INTEGRAL Workshop, ed. A. Gimenez, V. Reglero, \& C. Winkler, ESA SP-459, 191

Erber, T. 1966, Rev. Mod. Phys., 38, 626

Fierro, J. M., Michelson, P. F., Nolan, P. L., \& Thompson, D. J. 1998, ApJ, 494, 734

Gangadhara, R. T., \& Gupta, Y. 2001, ApJ, 555, 31

Gil, J., Melikidze, G. I., \& Mitra, D. 2002, A\&A, submitted

Harding, A. K. 2001, Gamma-Ray Pulsars: Models and Predictions, in Inter. Symp. on High Energy Gamma-Ray Astronomy, ed. F. A. Aharonian, \& H. J. Völk, AIP Conf. Proc., 558, 115

Harding, A. K., Baring, M. G., \& Gonthier, P. L. 1997, ApJ, 476, 246

Harding, A. K., \& Muslimov, A. G. 1998, ApJ, 508, 328

Harding, A. K., Tademaru, E., \& Esposito, L. W. 1978, ApJ, 225, 226

Kanbach, G., Bennet, K., Bignami, G. F., et al. 1980, A\&A, 90, 163

Kanbach, G., Arzoumanian, Z., Bertsch, D. L., et al. 1994, A\&A, 289, 855

Kanbach, G. 1999, Temporal and spectral details of bright EGRET pulsars, in Proc. of the Third INTEGRAL Workshop, ed. A. Bazzano, G. G. C. Palumbo, \& C. Winkler, Astrophys. Lett. Comm., 38, 17

Kuiper, L., Hermsen, W., Verbunt, F., et al. 2000, A\&A, 359, 615

Miyazaki, J., \& Takahara, F. 1997, MNRAS, 290, 49

Morini, M. 1983, MNRAS, 202, 495

Rudak, B., \& Dyks, J. 1999, MNRAS, 303, 477

Rudak, B., \& Ritter, H. 1994, MNRAS, 267, 513

Schönfelder, V., Bennett, K., Blom, J. J., et al. 2000, A\&AS, 143, 145

Sturrock, P. A. 1971, ApJ, 164, 529

Thompson, D. J. 2001, Gamma-Ray Pulsars: Observations, in Inter. Symp. on High Energy Gamma-Ray Astronomy, ed. F. A. Aharonian, \& H. J. Völk, AIP Conf. Proc., 558, 103

Thompson, D. J., Harding, A. K., Hermsen, W., \& Ulmer, M. P. 1997, in Proc. of the 4th Compton Symposium, ed. C. D. Dermer, M. S. Strickman, \& J. D. Kurfess, AIP, 410, 39

Zheng, Z., Zhang, B., \& Qiao, G. J. 1998, A\&A, 334, L49 